J. Perinat. Med. 30 (2002) 242-249

\section{The midwife factor in obstetric procedures and neonatal outcome}

\author{
Peter Konstantiniuk ${ }^{1}$, Ilse Kern ${ }^{2}$, Albrecht Giuliani ${ }^{3}$, and Franz Kainer ${ }^{4}$
}

${ }^{1}$ Second Department of Surgery, LKH-Graz, ${ }^{2}$ Department of Internal Medicine, University of Graz, ${ }^{3}$ Department of Obstetrics and Gynecology, University of Graz, Austria, and ${ }^{4}$ First Gynecological Hospital, University of Munich, Munich, Germany

\section{Introduction}

Changing Childbirth [3], a report of the Department of Health, has prompted considerable debate about the future of the maternity services. Since maternity care provided by midwives seems to be as safe as that provided by doctors and women prefer it [19], midwifery-led-care has been introduced at several centers [11]. There are significant differences in the way that midwives and obstetricians view these changes [20], e.g. $56 \%$ of obstetricians but $81 \%$ of midwives prefer midwives being independent practitioners with their own case loads; $72 \%$ of obstetricians and $86 \%$ of midwives wish obstetricians to no longer see low-risk women with normal pregnancies, $91 \%$ of obstetricians and $92 \%$ of midwives wish more continuity of care during the antenatal period, which has a beneficial effect on both medical outcomes and maternal satisfaction [6]. All these changes would lead to a more extensive involvement of midwives in maternal care.

In the face of this tendency towards midwiferyled-care, it was our purpose to examine the extent of the midwife's influence in a University hospital and to determine whether this influence was due to length of experience. Although the study situation in Austria is quite different from Great Britain (midwives have a lower educational level, and doctors are always present at delivery), we expected some correlation.

The role of the midwife can be examined with two different perspectives, firstly as a group of Health
Care Providers (this point of view is discussed elsewhere $[2,16,18,22]$, secondly as individual persons with respect to different practice styles and different personal characteristics, which is the main subject of this study.

Interindividual differences of midwives have only been reported for episiotomy rates $[9,24]$ and counseling results [14]. One focus of our study was to determine the effect of episiotomy rates on neonatal outcome. Do newborns delivered by midwives with high episiotomy rates fare better than newborns delivered by midwives with low episiotomy rates?

Interindividual differences of cesarean section rates, forceps rates and neonatal outcome have only been studied for obstetricians $[1,4,7,18]$ but not for midwives. A primary aim of this study is to fill this gap.

\section{Methods}

\subsection{Data}

5384 deliveries at the Department of Obstetrics and Gynecology, University of Graz, Austria, from $1^{\text {st }}$ July 1993 to $31^{\text {st }}$ December 1994 were investigated. The following data were collected continuously over the whole observation period with a computer assisted database: mode of delivery, $\mathrm{pH}$ of umbilical artery, Apgar score, birth weight, gestational age, maternal age, identity and age of the midwife, stillbirths and deliveries before arrival. 


\subsection{Organizational structure}

The Department of Obstetrics and Gynecology in Graz is a consultant led care unit with staff midwives. All together there were 31 midwives working in four groups, each one of them with an eight day shift rhythm consisting of 12 hour shifts:

dayshift / nightshift / off / dayshift / nightshift / off / off / off.

In each shift there were four midwives, one consultant obstetrician and three obstetrics residents in different levels of training. The first-year obstetrics resident (= Senior House Officer) was continuously present at the delivery ward, the other obstetricians just on request. There were seven labor rooms, one midwife was responsible for organizational matters (e.g. phoning, bleeping, writing the delivery log book) and had to attend one labor room, each other midwife had to attend two labor rooms. The distribution of laboring women to the midwives accorded strictly to the order of appearance (therefore quasi randomized) in the delivery ward. Shifts of obstetricians were 24 hour shifts and the shift roster was completely independent of the midwives' shift roster. The obstetrics resident in charge of labor and delivery was present on routine rounds or at least at the end of the second stage of labor and performed an episiotomy either as a prophylactic episiotomy (rigid perineum or impending perineal tear) on the midwife's request or to shorten labor (e.g. impending fetal asphyxia) according to his/her own judgment. This resident was responsible for the management of the parturient women and for the documentation of the delivery characteristics. In case of any problem he/she had to call for a more experienced resident or for the on-call consultant obstetrician, who was the only person authorized to decide to proceed with an instrumental delivery (cesarean section or forceps: the only vaginal operative technique performed). The most experienced obstetrician present was responsible for the diagnosis.

Additionally, the resident was responsible for the newborn including performing the Apgar score. In the case of any existing or impending problem he/she had to call for the pediatrician. If present the pediatrician performed the Apgar score.

Continuous electronic fetal monitoring was used in laboring women whenever possible (99\%). Umbil- ical arterial blood gas studies on newborn infants were obtained routinely. Attending time was defined as time between arrival at the delivery ward and delivery. Spontaneous delivery was defined as vaginal delivery with cephalic presentation without forceps. All patients were attended by staff.

\subsection{General exclusions}

Six midwives who had attended less than one third of the count of the midwife with most deliveries (298) were excluded from all sections of the study $\left(\mathrm{n}_{1}=22, \mathrm{n}_{2}=31, \mathrm{n}_{3}=42, \mathrm{n}_{4}=50, \mathrm{n}_{5}=74, \mathrm{n}_{6}=89\right)$. Thus a count of 5076 deliveries remained for the examinations.

\subsection{Statistical approach}

Statistical analysis was performed with SPSS (Statistical Package for Social Scientists), p-values below 0.05 were called significant, below 0.005 highly significant, all p-values are two-tailed. Maternal age, mean gestational age at delivery and mean birth weights were compared with ANOVA and did not significantly vary among midwives.

The influence of the individual midwives on the mode of delivery (episiotomy rate, cesarean section rate, forceps rate) was analyzed with the chisquare-test. The influence of the individual midwives on $\mathrm{pH}$ of umbilical arteries was calculated with ANOVA, the effect on Apgar scores was calculated with the Kruskal-Wallis-test.

To control for the effect of the midwife's experience, we performed linear regression analysis, using the age of the midwife as the independent variable. This is valid, since age and experience are highly correlated [1] $(r=0.90 ; p=0.0001)$. If the dependent variable was of binary type (episiotomy rates, cesarean section rates, forceps rates) we used logistic regression analysis.

To study the effect of the individual practice style we performed (logistic) regression analysis using the individual episiotomy rates as the independent variable.

The Students' t-test was carried out to assess the relationship between the continuous variable $\mathrm{pH}$ and midwives-groups, Wilcoxon-rank-sum-test (corrected for ties) was used for the relationship between Apgar scores and midwives' groups. 


\section{Results}

\subsection{Episiotomy rates}

Included were all spontaneous deliveries except deliveries before arrival. The individual episiotomy rates of the different midwives (figure 1) ranged between a minimum of $31.6 \%$ and a maximum of $76.9 \%(\mathrm{p}<0.001 ; \mathrm{n}=4202$; overall mean $=$ $55.4 \%)$.

There was no evidence $(\mathrm{p}=0.16)$ for a linear relationship between age and episiotomy rates. We then performed regression analysis for outcome factors on episiotomy rates. The One-minute APGAR score $(p=0.97)$ and the Five-minutes APGAR score $(p=0,73)$ did not depend on episiotomy rates, but $\mathrm{pH}$ of umbilical arteries was positively correlated: intercept: $7.202(\mathrm{p}<0.001)$; episiotomy rate: $0.067(\mathrm{p}<0.001)$, which means, for example, that for an assumed episiotomy rate of $40 \%$ the expected ph would be 7.228 , for an assumed episiotomy rate of $60 \% 7.242$.

\subsection{Instrumental deliveries}

Included were all deliveries with an attending time of at least 45 minutes, stillbirths were excluded thus leaving a count of 4407 deliveries. The individual cesarean section rates of the different midwives (figure 2) ranged from a minimum of $6.3 \%$ to a maximum of $17.8 \%(\mathrm{p}=0.09$, overall mean $=$ $10.8 \%$ ); the individual forceps rates (figure 3 ) between a minimum of $1.7 \%$ and a maximum of $11.1 \%(\mathrm{p}=0.002$, overall mean $=5.4 \%)$. Age of midwives was not correlated with cesarean section frequencies $(\mathrm{p}=0.55)$ nor with forceps frequencies $(\mathrm{p}=0.59)$.

\subsection{Perinatal outcome}

Included were all deliveries with an attending time of at least 45 minutes and a birthweight of at least 1000 g except stillbirths, thus totaling 4318 deliveries.

pH of umbilical arteries. Figure 4 shows the blood gas samples taken by the different midwives, the individual means for $\mathrm{pH}$ of umbilical arteries ranging between a minimum of 7.21 and a maximum of $7.28(\mathrm{p}<0.001)$, overall mean: 7.236. Age was positively correlated with $\mathrm{pH}$ : intercept: 7.218 $(\mathrm{p}<0.001)$; age: $0.00049(\mathrm{p}<0.001)$.

Apgar scores. One-minute Apgar score: The overall mean was 8.67 ; the individual means ranged

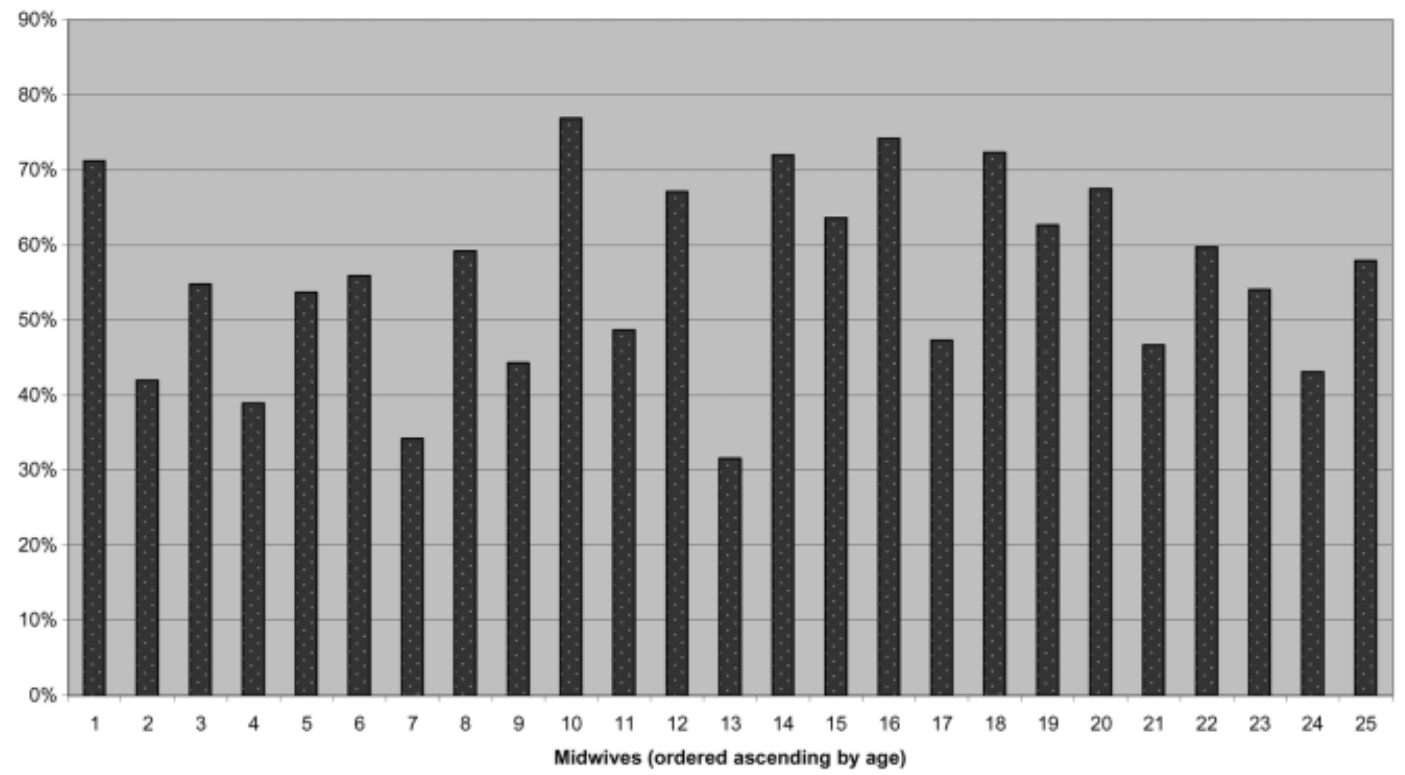

Figure 1. Episiotomy rates according to the different midwives. 


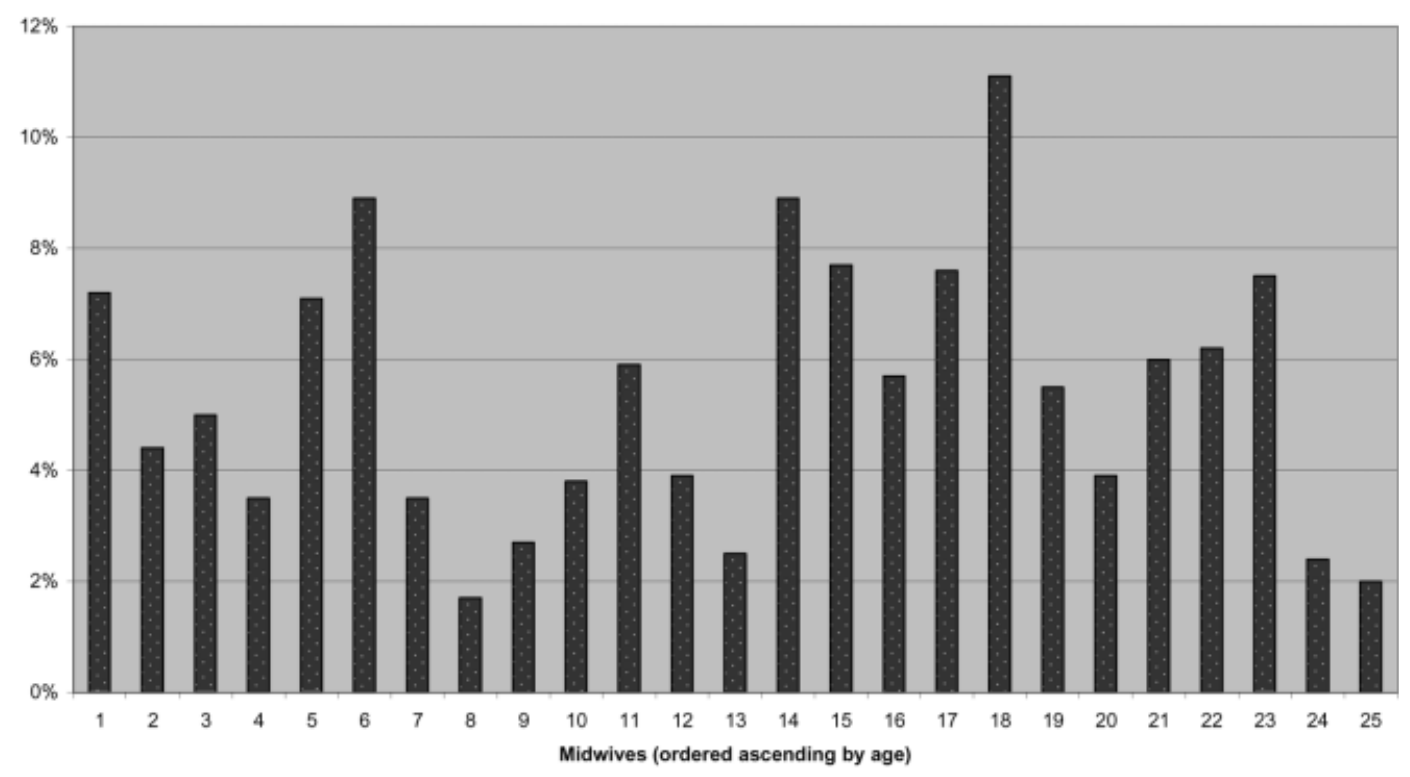

Figure 2. Cesarean section according to the different midwives.

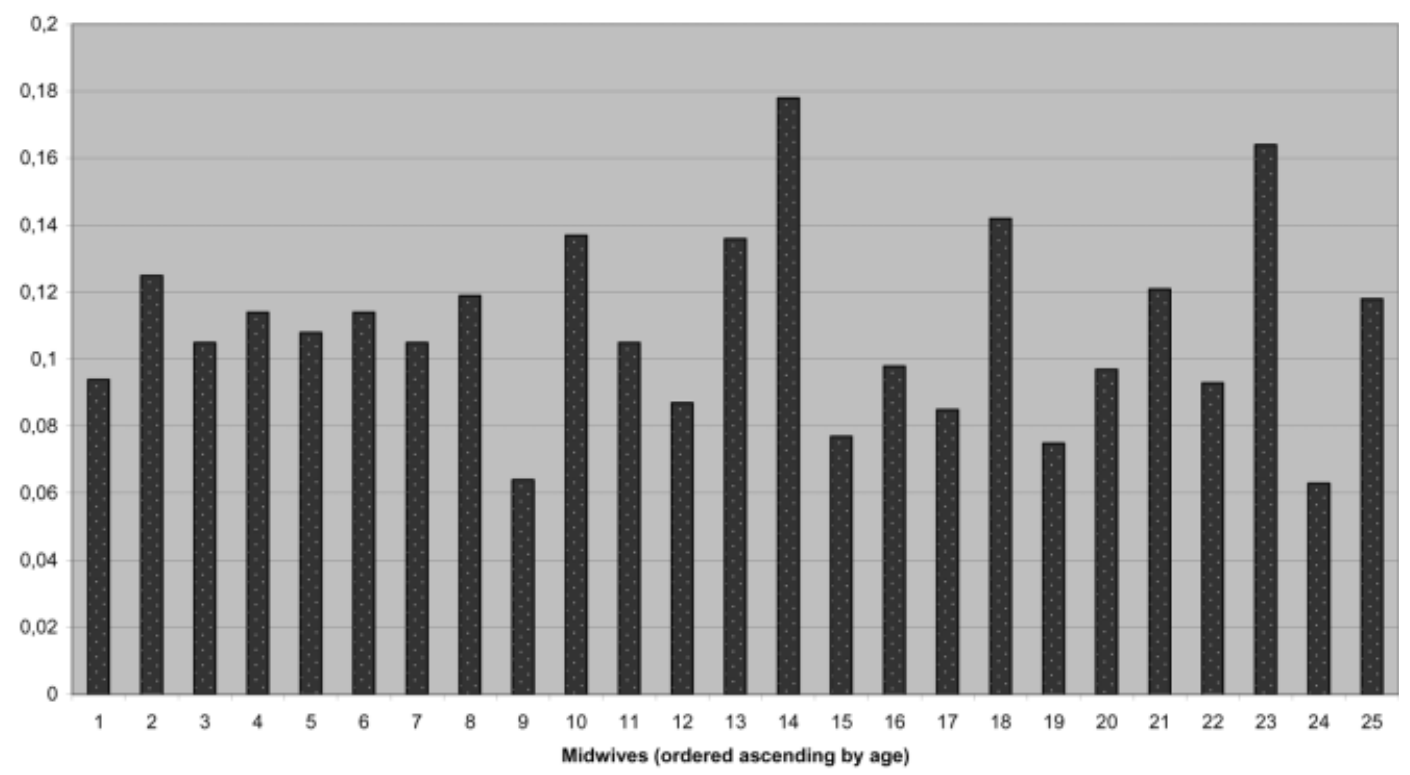

Figure 3. Forceps rates according to the different midwives.

from a minimum of 8.53 to a maximum of 8.86 $(\mathrm{p}=0.15)$. The one-minute Apgar score was significantly correlated with the age of the midwives (intercept: $8.5821 ; \mathrm{p}<0.001$; age: $0.00456 ; \mathrm{p}=0.009)$.
Five-minutes Apgar score. The overall mean was 9.88; the individual means ranged between a minimum of 9.67 and a maximum of 9.95 $(\mathrm{p}=0.29)$. The five-minutes Apgar score was not 


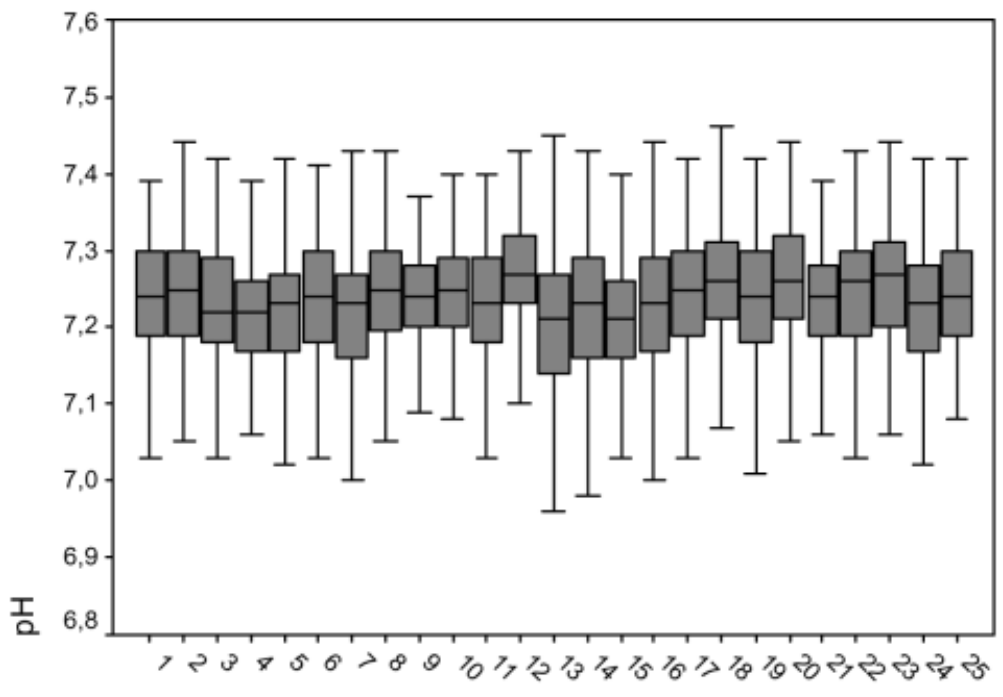

Midwives (ordered ascending by age)
Figure 4. Umbilical arteries blood gas samples according to the different midwives. significantly correlated with the age of the midwives $(\mathrm{p}=0.07)$.

\section{Discussion}

\subsection{Episiotomy}

Episiotomy rates varied markedly among midwives, which was comparable with earlier findings $[9,24]$. Our data do not permit us to distinguish whether episiotomy is overused by some or underused by others, but provision of health care should be primarily determined by need and not by the personal characteristics of the health care provider. Thus consensus should be found which might be an ideal episiotomy rate, i.e. at least each individual hospital should have a clear policy to reduce differences that are due just to personal characteristics. For example, Henriksen at al. [9] suggested an ideal episiotomy rate of about $20 \%$.

The associated question is, does a clear policy change anything? When Sleep et al. [21] allocated women to one of two perineal management policies, in one the aim was to restrict episiotomy to fetal indications; in the other the operation was to be used more liberally to prevent perineal tears. The resultant episiotomy rates were $10 \%$ and $51 \%$ respectively. The overall episiotomy rate in spontaneous vaginal deliveries was $61 \%$ before the trial and decreased to $31 \%$ during the trial. Interestingly it again decreased to $20 \%$ after the trial, when no target rate was set anymore. This means, that even focusing on a subject alone influences routine management - even more so if a certain task is given. Defining such a task is effective, which was shown in the last two decades, when the cesarean section rate had dramatically increased and, after consensus shown at conferences and in many articles, decreased again (e.g. cesarean section rates of the University Hospital of Graz: $7.1 \%$ in 1980, $13.4 \%$ in $1985 ; 9.4 \%$ in 1995$)$.

Postulating an effect due to amount of experience, there should be a linear relationship between episiotomy frequencies and age of the midwives. This idea was refuted by our results, which indicate that the major determinant of the use of episiotomy was the individual practice style of each midwife.

Comparing midwives with different episiotomy rates, we found a dependence for ph of umbilical arteries being statistically significant but quantitatively small. Taking into account the fact that Apgar scores did not vary significantly, we believe the extent of this difference to be negligible. Therefore the midwives with low episiotomy rates do not place infants at greater risk by the avoidance of many episiotomies in their patient population. 


\subsection{Cesarean section}

Cesarean section rates did not vary significantly among midwives and were not correlated with the midwife's age, which reflects the fact that the decision for cesarean section is taken by a consultant obstetrician and therefore can be assumed to be independent of midwives.

\subsection{Forceps and neonatal outcome}

A correlation with the midwives' age was found for blood gas samples and one-minute Apgar score, but not for forceps rates and five minutes Apgar score. Forceps rates as well as blood gas samples but not Apgar scores varied significantly among midwives, which was quite surprising since the decision for any kind of treatment is taken by the obstetrician i.e. the decision for a forceps delivery by the consultant obstetrician.

How could the midwife influence forceps rates and neonatal outcomes? There are three possible routes of influence for the midwives: the relationship between the midwife and the parturient woman, the midwife herself; and the relationship between the midwife and the obstetrician.

(a) Relationship between the midwife and the parturient woman Pain management. Wakefield [25] reported, that nurses tended to categorize patients according to symptoms or overt pain behavior. This essentially resulted in patients not being believed when they signaled that pain was becoming a distressing symptom. Pain perception is influenced either in a positive or negative direction by the individual's cognitive and psychological processes. We believe these findings to be transferable from nurses to midwives, but the main routes regarding pain and pain management in the relationship-triangle of patient-midwife-obstetrician are not clear and have not been investigated.

Emotional support decreases forceps frequencies $[10,23]$ and involves trust, which has been shown to be an essential component of nursing practice [17] and hence we believe for midwifery practice too.

Anxiety increases levels of catecholamines [12], which decrease uterine contractility [26]. If the relationship becomes therapeutic the midwife is able to lessen maternal anxiety, which consecutively re- duces catecholamine levels and facilitates uterine contractile activity and uterine blood flow and shortens the duration of labor. Bearing in mind that it is common practice to use forceps after two hours of the second stage of labor, this might be the crucial point to increase or decrease forceps delivery rates.

McCrea et al. [13] studied the relationship of the midwives with their clients by using a qualitative approach and were able to identify four main issues as influential in the development of therapeutic relationships: the nature and value of the midwives' role; recognition of authority/autonomy in practicing this role; emotional involvement with clients; and maintaining personal integrity.

A quantitative assessment including both the midwives' and the clients' characteristics is needed to show the extent to which the midwife/client relationship influences the use of obstetric procedures and neonatal outcome, i.e. does a 'poor' relationship cause harm?

(b) The midwife herself, her educational standard and her professional skills might be influential. The linear relationship between midwife age and $\mathrm{pH}$ is significant, but the extent is low, e.g. for age $=20$ the estimated ph is 7.228, and for age $=60$ the estimated $\mathrm{pH}$ is 7.247 , but this might indicate that older - and therefore more experienced midwives - have better strategies in birth management. On the other hand the interindividual variation shows a larger extent (7.21 vs. 7.28). DeMott [4] argues, that individual rates among obstetricians vary with anticipated risk of litigation. However, we believe this to be unimportant for the midwives of our study, since litigation is still a rare occurrence (less than one case per year) at the University Hospital of Graz.

(c) The relationships between midwives and obstetricians have traditionally been antagonistic [5], and characterized by inter-professional rivalries [15] but no attention has been given to the effect on obstetric interventions and neonatal outcome. The midwife is likely to be the first person to detect abnormalities in labor and it is up to her to decide when to call for the obstetrician. The obstetrician is responsible for the parturient women and formally takes the decision for any kind of medical treatment (like oxytocin, epidurals) but he/she is to some extent influenced by the midwife. Another 
study is needed to investigate the midwife-obstetrician-relationship and the extent to which it influences intervention rates and neonatal outcome.

Although a retrospective study, the present study model had the advantage that patients and midwives were not aware that they were participating in a study at the time of delivery. This is important, since a population being studied changes its be-

\begin{abstract}
Aims. In the face of major tendency towards midwiferyled-care it was our purpose to investigate the extent of the influence of the midwife on the rates of obstetric procedures and perinatal outcome.

Methods. 5384 consecutive deliveries at the Department of Obstetrics and Gynecology, University of Graz, were enrolled in the study. The following data were collected: mode of delivery, $\mathrm{pH}$ of umbilical artery, Apgar score. Firstly, data were investigated for interindividual differences and, secondly, for relationship with age of the midwife as a measure of experience.

Results. Interindividual differences were significant for episiotomy rates (minimum: $31.6 \%$; maximum $76.9 \%$; $\mathrm{p}<0.001$ ), forceps rates (minimum: $1.7 \%$; maximum $11.1 \% ; \mathrm{p}=0.002$ ) and $\mathrm{pH}$ of umbilical arteries (minimum:
\end{abstract}

havior, as happened at the West Berkshire perineal management trial [21] and is known as the Hawthorne effect [8] - and therefore results may be biased.

In general whenever planning or analyzing a study, more attention should be given to the individual personal influence as an underestimated factor in medical procedures.

7.21; maximum: 7.28; $\mathrm{p}=0.001$ ) but not for cesarean section rates and Apgar scores.

Linear regression analysis was significant between age of midwives and $\mathrm{pH}$ of umbilical arteries $(\mathrm{p}<0.001$; $\mathrm{r}=0.055)$ and for one-minute Apgar score $(\mathrm{p}=0.009$; $r=0.050$ ) but not for episiotomy rates, cesarean section rates, forceps rates and five-minutes Apgar score.

Conclusions. There are large interindividual differences in obstetric intervention rates which cannot be explained by the midwives' age. Provision of health care should be primarily determined by need and not by the personal characteristics of the health care provider, thus interindividual differences should be reduced and more often taken into account when analyzing any kind of data.

Keywords: Apgar score, Austria, blood gas analysis, cesarean section, episiotomy, forceps, individuality, midwifery, umbilical arteries.

Acknowledgement. We like to thank Professor Charles Rodeck, Dr. Ursula Pueringer and Mr. Kismet HossainIbrahim for the critical review.

\section{References}

[1] Berkowitz GS, GS Fiarman, MA Mojica, J Bauman, RH de Regt: Effect of the physician characteristics on the cesarean birth rate. Am J Obstet Gynecol 161 (1989) 146

[2] Butler J, B Abrams, J Parker, JM Roberts, RK Laros: Supportive nurse-midwife care is associated with ad reduced incidence of cesarean section. Am J Obstet Gynecol 168 (1993) 1407

[3] Changing childbirth: Report of the Expert Maternity Group. Department of Health. London, HMSO, 1993

[4] DeMott RK, HF Sandmire:The Green Bay cesarean section study I: the physician factor as a determinant of cesarean birth rates. Am J Obstet Gynecol 162 (1990) 1593

[5] Donnison J: Midwives and medical men: a history of the struggle for the control of childbirth. London: Historical Publications $2 / 1988$

[6] Flinc C, P Puoengeris, AM Grant: The 'Know Your
Midwife' scheme: a randomised trial of continuity of care by a team of midwives. Midwifery 5 (1989) 11

[7] Goyert GL, SF Bottoms, MC Treadwell, PC Nehra: The physician factor in cesarean birth rates. N Engl J Med 320 (1989) 706

[8] Handy C: The Hawthorne studies. In: Charles Handy: Understanding Organisations. London, Penguin Books $4 / 1993$, pp 156

[9] Henriksen TB, KM Bek, M Hedegaard, NJ Secher: Episiotomy and perineal lesions in spontaneous vaginal deliveries. Br J Obstet Gynaecol 99 (1992) 950

[10] Kennell J, M Klaus, S McGrath, S Robertson, C Hinkley: Continuous emotional support during labor in a United States hospital. A randomized controlled trial. JAMA 265 (1991) 2197

[11] Lachelin GCL: Midwifery Led Care Meeting. Newsletter of the Department of Obstetrics and Gynecology. London, UCL Medical School 1996 
[12] Ledermann RP, E Lederman, BA Work Jr, DS McCann: The relationship of maternal anxiety, plasma catecholamines, and plasma cortisol to progress in labor. Am J Obstet Gynecol 132 (1978) 495

[13] McCrea H, V Crute: Midwife/client relationship: midwives' perspectives. Midwifery 7 (1991) 183

[14] Meadows J, S Jenkinson, J Catalan, B Gazzard: Voluntary HIV testing in the antenatal clinic: differing uptake rates for individual counselling midwives. Aids-Care 2 (1990) 229

[15] Oakley A: The origins and development of antenatal care. In: Enkin M, I Chalmers (eds): Effectiveness and satisfaction in antenatal care. London, Heinemann 1982

[16] Oakley D, T Murtland, F Mayes, R Hayashi, BA Petersen, C Rorie, F Andersen: Processes of care. Comparisons of certified nurse-midwives and obstetricians. J Nurse Midwifery 40 (1995) 399

[17] Pask EJ: Trust: an essential component of nursing practice-implications for nurse education. NurseEduc-Today 15 (1995) 190

[18] Pel M, MHB Heres, AAM Hart, F van der Veen, PE Treffers: Provider-associated factors in obstetric interventions. Eur J Obstet Gynecol Reprod Biol 61 (1995) 129

[19] Renfrew MJ: Midwife vs. medical/shared care. In: Enkin MW, MJNC Keirse, MJ Renfrew et al. (eds.): Pregnancy and childbirth module, Cochrane database of systematic reviews. Review no. 03295, Cochrane updates on disk, disk issue 1. Update Software, Oxford 1994

[20] Sikorski J, S Clement, JWilson, S Das, N Smeeton:A survey of health professionals' views on possible changes in the provision and organisation of antenatal care. Midwifery (1995) 61

[21] Sleep J, A Grant, J Garcia, D Elbourne, J Spencer, I Chalmers: West Berkshire perineal management trial. Br Med J 289 (1984) 587

[22] Slome C, H Wetherbee, M Daly, K Christensen, M Meglen, H Thiede: Effectiveness of certified nursemidwives: a prospective evaluation study. Am J Obstet Gynecol 124 (1976) 177

[23] Sosa R, J Kennell, M Klaus, S Robertson, J Urrutia: The effect of a supportive companion on perinatal problems, length of labor, and mother-infant interaction. N Engl J Med 303 (1980) 597

[24] Thranov I, AM Kringelbach, E Melchio, O Olsen, MT Damsgaard: Postpartum symptoms. Episiotomy or tear at vaginal delivery. Acta Obstet Gynecol Scand 69 (1990) 11

[25] Wakefield AB: Pain: an account of nurses' talk. J Adv Nurs 21 (1995) 905

[26] Zuspan FP, LA Cibils, SV Pose: Myometrial and cardiovascular responses to alterations in plasma epinephrine and norepinephrine. Am J Obstet Gynecol 84 (1962) 841

Received May 8,2001. Revised September 13,2001. Accepted October 6,2001.

Peter Konstantiniuk, M.D.

$2^{\text {nd }}$ Department of Surgery

Auenbrugger Platz 5

8010 Graz

Austria

Tel.: +4331638581315 